\title{
Super-resolving multiple scatterers detection in synthetic aperture radar tomography assisted by correlation information (Erratum)
}

\author{
Ahmad Naghavi, ${ }^{\text {a,* }}$ Mohammad Sadegh Fazel, ${ }^{\text {a }}$ Mojtaba Beheshti, ${ }^{\text {b }}$ and \\ Ehsan Yazdian ${ }^{\text {a }}$ \\ asfahan University of Technology, Department of Electrical and Computer Engineering, \\ Isfahan, Iran \\ ${ }^{\mathrm{b}}$ Isfahan University of Technology, Research Institute for Information and Communication
} Technology, Isfahan, Iran

[DOI: $10.1117 / 1 . J R S .15 .039901]$

This article [J. of Applied Remote Sensing, 14(3), 034517 (2020) 10.1117/1.JRS.14.034517] was originally published with an error in Fig. 1. Specifically, \rho_s was incorrect in the original version of Fig. 1:

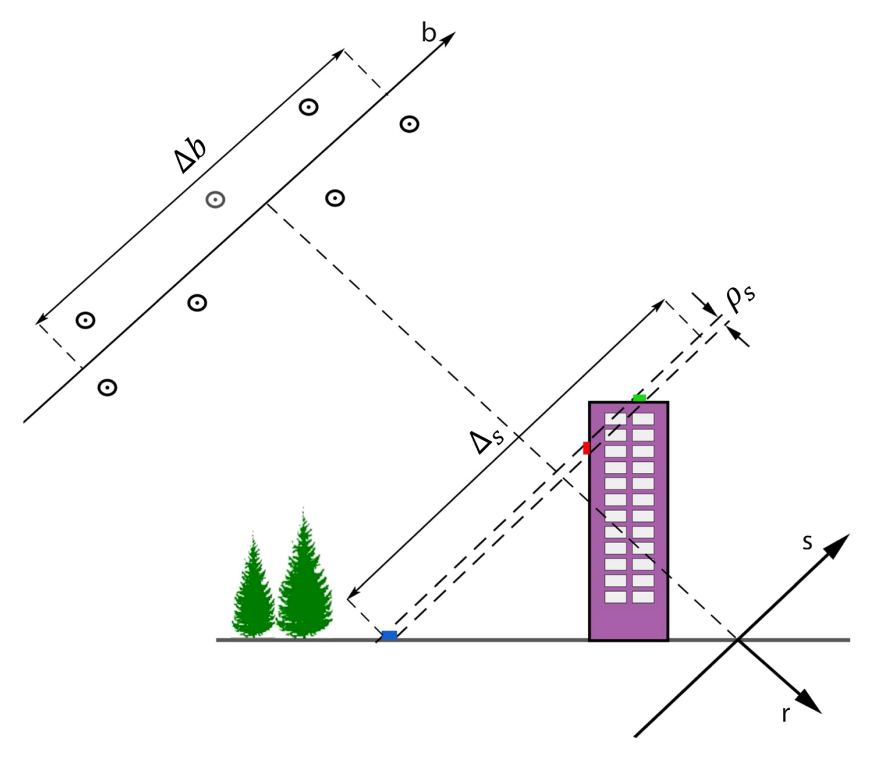

*Address all correspondence to Ahmad Naghavi, a.naghavi@ec.iut.ac.ir 
The corrected version includes \rho_r:

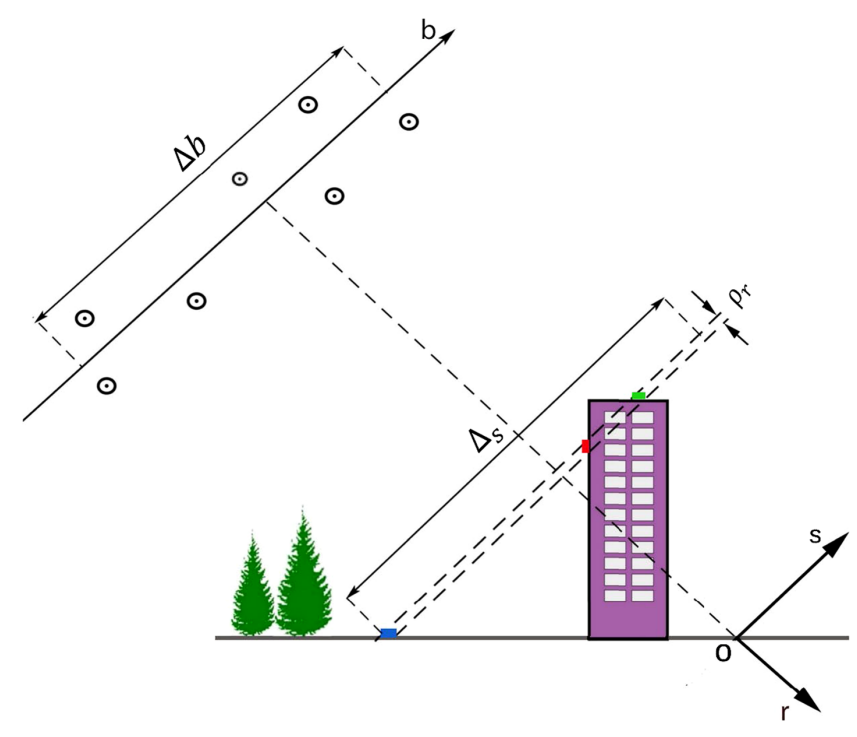

The original caption for the figure is correct and remains unchanged.

The original image was replaced with the corrected version and the article was republished on 02 July 2021. 